\title{
Pollution, colonialité et droit international de l'environnement : Une étude de la situation palestinienne
}

Inquinamento, colonialità e diritto internazionale dell'ambiente: uno studio della situazione palestinese

Pollution, Coloniality and Environment International Law: A Study of Palestinian Situation

Joni Aasi

\section{OpenEdition}

Journals

Édition électronique

URL : https://journals.openedition.org/aam/3870

DOI : $10.4000 /$ aam.3870

ISSN : 2038-3215

Éditeur

Dipartimento Culture e Società - Università di Palermo

Référence électronique

Joni Aasi, «Pollution, colonialité et droit international de l'environnement : Une étude de la situation palestinienne », Archivio antropologico mediterraneo [En ligne], Anno XXIV, n. 23 (1) | 2021, mis en ligne le 30 juin 2021, consulté le 02 juillet 2021. URL : http://journals.openedition.org/aam/3870 ; DOI : https://doi.org/10.4000/aam.3870

Ce document a été généré automatiquement le 2 juillet 2021.

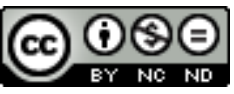

Archivio antropologico mediterraneo è distribuita con Licenza Creative Commons Attribuzione - Non commerciale - Non opere derivate 4.0 Internazionale. 


\title{
Pollution, colonialité et droit international de l'environnement : Une étude de la situation palestinienne
}

\author{
Inquinamento, colonialità e diritto internazionale dell'ambiente: uno studio della \\ situazione palestinese
}

Pollution, Coloniality and Environment International Law: A Study of Palestinian Situation

Joni Aasi

\section{Introduction}

1 En octobre 2019, la première pierre a été posée en vue de la construction d'une zone de recyclage et de traitement des déchets sur un terrain adjacent à la colonie de Ma'ale Adumim, à l'est de Jérusalem (Heller 2019). Ce parc industriel absorbera les déchets de la ville de Jérusalem, mais aussi les déchets venant de l'ensemble d'Israël. Bien entendu, ce n'est pas la première usine du genre en Cisjordanie, il existe des dizaines d'usines traitant des substances dangereuses et toxiques.

D'après Mosi Raz, qui fut un membre de la Knesset, élu du parti de gauche Meretz, le but de ce projet est de justifier le transfert de ressources financières aux colonies et de renforcer la mainmise israélienne sur les Territoires palestiniens occupés (Berger, Tzafrir 2018). Il paraît clair que ce parc industriel fait partie de la politique israélienne d'expansion coloniale. En 1996, la Haute Cour de Justice d'Israël a autorisé l'extension de la colonie de Maale Adumim au détriment des communautés bédouines vivant sur les terres concernées, accordant ainsi une légitimité et une base légale à leur transfert (HCJ 2966/95, 28/05/1996). En 1999, le gouvernement israélien a approuvé le plan E1 qui donne plus de terres à la colonie de Maale Adumim (une zone de 1200 hectares 
destinée à créer un bloc urbain entre Jérusalem et Maale Adumim). Entre 1997 et 2007, plus de 150 familles des communautés bédouines ont été expulsées de la région de Maale Adumim selon l'association israélienne de défense des droits de l'homme, B'Tselem. Le rapport de B'Tselem conclut que " l'Administration civile envisage d'expulser les communautés bédouines résidant actuellement dans cette région ».

Certains colons s'opposent à la création de cette zone de traitement des déchets à cet endroit mais leur position tient plutôt de ce que l'on peut décrire comme un écologisme colonial, associé à une mode de consommation de la société post-industrielle. Cet écologisme est à distinguer de celui défendu par les Palestiniens et en particulier les Bédouins qui sont les plus touchés par ce projet qui relève d'un écologisme indigène ou " écologisme des pauvres " dont les origines se trouvent dans le conflit sur les ressources naturelles et la lutte contre leur pillage (Guha, Martinez-Alier 1997 : 17-18). L'une des communautés bédouines vivant dans la région est la communauté de Wadi Saab qui compte 24 familles ; 14 familles sont stables, vivent là toute l'année tandis que dix familles se déplacent ailleurs selon les saisons à la recherche de pâturages. Toutes les familles vivent à la fois de l'élevage de chèvres et de moutons et d'emplois dans la colonie de Maale Adumim. La communauté de Wadi Saab est originaire de la région de Beer Shevaa (Naqab). Ils ont été expulsés de leur région pendant la guerre qui a présidé à la création de l'Etat d'Israël en 1948. Leur nakba (catastrophe) se poursuit car ils sont menacés d'être, de nouveau, expulsés de Wadi Saab. Après l'occupation israélienne des Territoires palestiniens en 1967, ils se sont à nouveau retrouvés confrontés aux pressions de l'armée et des colons en vue de les faire partir. Ces pressions sont devenues au cours des dernières décennies de plus en plus intenses.

Aborder ensemble les questions de la pollution et de la colonisation, c'est privilégier la dimension historique et sociale du conflit. Récemment, des historiens tels que Jacques Pouchepadass et Karin Mickelson se sont intéressés au lien entre environnement et fait colonial. Karin Mickelson a souligné l'absence d'histoire environnementale prenant en compte la contribution de l'expérience coloniale à la détérioration de l'environnement mondial. Elle évoquait la position de l'historien de l'environnement, Richard Grove, qui déclarait : « C'est dans les colonies tropicales, que les scientifiques ont réalisé pour la première fois la vitesse étonnante à laquelle les peuples, en particulier les Européens, pouvaient transformer ou même détruire l'environnement naturel ". Pour elle, l'histoire environnementale devrait s'attacher à mettre en évidence la contradiction entre impérialisme et protection de l'environnement (Mickelson 2013 : 57). Il est à souligner qu'au XIXe siècle, les termes d'impérialisme et du colonialisme (Gathii 2006-2007 : 1014) impliquent une conception des inégalités dépassant de loin le domaine économique. Ces termes peuvent s'appliquer aussi sur la distribution inégale de la pollution comme par exemple le transfert d'une usine très dangereuse de la ville israélienne Kfar Saba à l'autre côté de la ligne verte ${ }^{1}$, à la ville palestinienne de Tulkarm².

5 La dichotomie modernité/colonialité distinguée par le penseur péruvien, Anibal Quijano, me paraît très utile pour comprendre la politique coloniale de domination imposée aux Palestiniens et pour souligner que la formation de l'Etat d'Israël a dépendu d'un processus de colonisation. La colonialité renvoie à une matrice de forces qui comprend, outre le contrôle administratif, militaire et économique, des dispositifs de contrôle aussi dans le domaine de l'environnement, et dont le racisme est la manifestation la plus perceptible et la plus omniprésente de ces forces (Quijano 2007 : 
112). La modernité devient colonialité où "tout acte de vie, d'installation, d'extraction, de récolte ou de commerce devient la violence elle-même" (Weizman 2004). Et la modernité devient colonialité et "un paradigme de guerre" (Soto 2009 : 54) comme le constate le rapport de Human Rights Watch,"While Israeli settlements flourish, Palestinians under Israeli control live in a time warp - not just separate, not just unequal, but sometimes even pushed off their lands and out of their home"s.

Qui plus est, l'Etat-nation est, selon Anibal Quijano, une forme de la modernité qui émerge d'abord d'un processus de colonisation d'un peuple sur d'autres peuples et dépend d'une organisation centralisée de l'espace conquis (Quijano 2000 : 558). La formation de l'Etat d'Israël en est un exemple, elle s'inscrit dans un processus de colonisation dans la mesure où ont été mis en place toute une série de modalités de contrôle non seulement de l'économie et des statuts et droits mais aussi de tous les aspects de la vie des palestiniens.

7 Je propose donc d'analyser l'occupation coloniale israélienne, dont l'accélération et l'intensité caractérisent "le processus de paix", à partir du droit international de l'environnement : c'est-à-dire d'une part d'analyser les revendications des palestiniens dans ce domaine et, d'autre part la responsabilité de l'Etat israélien vis-à-vis de crimes environnementaux.

\section{Entre les droits égaux la force décide}

Dans le cadre des accords d'Oslo, la coopération institutionnelle dans le domaine de l'environnement a été développée. Tout d'abord, l'annexe III de la Déclaration de principes de 1993 prévoyait des programmes de développement et de coopération économique qui nécessitaient la création d'un comité mixte de coopération économique s'intéressant à de nombreuses questions, notamment aux questions environnementales liées à l'eau, à l'énergie et à l'industrie. Puis, l'article 12 des accords d'Oslo II prévoyait un transfert limité des pouvoirs en matière d'environnement de l'administration civile israélienne d'occupation à l'Autorité palestinienne. Le transfert de pouvoirs a été lié à la division des territoires palestiniens en trois zones A, B et C. Comme l'ont reconnu les responsables israéliens et palestiniens, Oslo a échoué en ce qui concerne les questions environnementales (Alleson et al. $2013: 16$ ).

9 La répartition des tâches et des responsabilités entre Israël et l'Autorité palestinienne rend difficile le respect du principe de " bonne gestion de l'environnement ». La responsabilité du traitement des déchets relève des responsabilités de l'Autorité palestinienne dans les zones A et B, alors que c'est la responsabilité des autorités d'occupation dans la zone $C$ (qui couvre $61 \%$ des Territoires palestiniens occupés). Non seulement cette division ne prend pas en compte les caractéristiques des questions environnementales qui nécessitent une politique unifiée pour l'ensemble du territoire occupé, mais elle permet à Israël d'utiliser la zone $C$ pour enterrer et traiter ses déchets. Il ne faut pas oublier que la division du territoire entre A, B et C, proposée par les Accords, fait référence à trois catégories, qui relèvent toutes des Territoires dits palestiniens occupés depuis 1967, et la différence entre eux réside uniquement dans les pouvoirs à transférer de l'État occupant à l'Autorité palestinienne. Ce transfert de pouvoirs est un processus graduel qui doit se faire en respectant le droit international et le droit à l'autodétermination du peuple palestinien comme vient de le rappeler tout récemment le Tribunal Pénal International. ${ }^{4}$ 
$10 \mathrm{Au}$ contraire, par ses interprétations des Accords, la Haute Cour de Justice israélienne (HCJ) a permis l'exploitation des ressources naturelles et l'utilisation des terres des Palestiniens pour traiter les déchets provenant d'Israël et de ses colonies. Ici, je veux m'arrêter sur deux points évoqués par l'ancienne présidente de la Haute Cour de Justice israélienne, la juge Dorit Beinich, dans la décision concernant les carrières israéliennes dans les territoires palestiniens occupés (HCJ 09/2164). Elle a tout d'abord souligné que les modalités d'exploitation des carrières dans la zone $\mathrm{C}$ dépendraient du calendrier de négociations entre Israël et les Palestiniens. Selon la troisième annexe de l'accord intérimaire (Oslo II), les pouvoirs d'octroi de licences, de supervision, de croissance et d'emploi devaient être progressivement transférés de l'administration civile à la partie palestinienne dans le cadre d'un processus global de transfert de pouvoirs et de responsabilités dans ces domaines. Puis, elle a ajouté que les deux parties ont convenu que pendant la période transitoire les carrières continueront de fonctionner : les questions soulevées lors du processus de transfert des droits seront discutées par un comité paritaire qui réunit les deux parties qui se sont engagées à respecter ses recommandations, et que "jusqu'à ce qu'une décision soit rendue par le comité mixte, la partie palestinienne ne prendra aucune mesure susceptible de nuire à ces carrières".

11 La parité entre Palestiniens et Israéliens est évoquée dans une thèse de doctorat en géographie de l'Université hébraïque sur "La gestion transnationale de l'environnement dans des conditions inégales", soutenu par Nitzan Levy. Selon ce dernier, les succès et les échecs de la coopération israélo-palestinienne en matière d'environnement sont comparables à d'autres situations tout en reconnaissant qu'il n'y a pas de frontières entre Israël et les Territoires palestiniens occupés (Levy 2009). Ce travail élude la question de l'occupation et du colonialisme. Des rapports internationaux font de même, en évacuant la question du conflit territorial et en demandant aux deux parties de coopérer pour faire face aux enjeux environnementaux relevés par les accords internationaux. Ces rapports soulignent les problèmes liés à l'environnement que rencontrent particulièrement les Palestiniens. Toutefois, au regard de la responsabilité de la situation environnementale, ils placent sur un quasipied d'égalité les deux parties en leur demandant de coopérer tout en considérant qu'Israël porte une plus grande responsabilité en raison de sa puissance et de son économie. ${ }^{5}$

Susan Marks a développé la notion d'exploitation en droit international : une notion qui se réfère à une structure où ce qui est réalisé par une partie est aux dépens de l'autre partie. Cette structure est masquée par une idéologie de la mutualité (Ideology of mutuality) au sens d'une responsabilité partagée. En termes marxiens, « entre les droits égaux la force décide" (Marks 2008 : 302-305). Cela correspond exactement à la coopération israélo-palestinienne au sein du comité mixte de l'environnement. Ce comité mixte est composé d'un même nombre de représentants de chaque partie, son processus de décision se fait par consensus, et par conséquent ses références premières restent les ordres militaires israéliens. Parmi ces ordres, on peut souligner en particulier les ordres militaires tels que l'ordre militaire (2) 1967, qui considère que l'eau est une propriété publique qui doit être contrôlée par l'État occupant et les ordres rendus au cours des années 1967/1968 concernant l'octroi de licences, la fixation de quotas et la déclaration de nullité de tous les règlements antérieurs. 


\section{La « colonisation toxique » en Palestine}

13 La logique coloniale per se de l'occupation israélienne est de plus en plus fréquemment évoquée par des auteurs comme Virginia Tilley

It [colonialism] is a foreign grab for land and resources (here including symbolic resources, such as sacred sites) enabled by superior military power. Thus, a colonial conflict is not resolved by getting people to 'see each other as human beings' but by addressing the basic injustice of foreign domination and restoring the people's capacity to exercise their right to self-determination. Similarly, an apartheid regime is not created out of 'misunderstanding', but to serve a group's survival agenda by physically excluding other groups-a policy that has little to do with the nature of others except that they are, by definition, others. A group that believes it cannot survive and flourish with others, and so must absolutely dominate others to preserve a life-world essential to its own cultural or physical survival, will remain concerned to maintain that domination no matter what others do or say or any underlying justice of their demands (Tilley 2011: 12).

14 Cette logique coloniale est analysée comme antérieure à 1967. Pour John Strawson, son origine date des années 1920 :

It's a colonial domination whose origins date back to the 1920's" (...)."The connection of the settlements to the settlement of the 1920's and 1930's in the mind of the Israeli regime legitimize each other. The case for the creation of the state of Israel was inextricably connected to the settlement of the land through the pioneers (the halutzim) who through their physical occupation provided the structure for Jewish immigration and the creation of a significant population, which claimed the right to self-determination (Strawson 2002: 371).

Dans une perspective similaire, le politologue américain John Collins utilise le terme d'occupation non pas au sens du droit humanitaire (la présence militaire d'un Etat dans un territoire appartenant à un autre peuple ou Etat) mais au sens sociologique en distinguant l'occupation colonialiste de peuplement (settler colonialism) de l'occupation des Palestiniens du territoire par le fait d'y habiter depuis des siècles (Collins 2010 : 199).

En présentant une symétrie entre palestiniens et israéliens les accords d'Oslo servent à occulter la domination israélienne et son colonialisme. Martin Heger qualifie du "colonialisme toxique" la nouvelle politique coloniale des pays européens qui ne vise plus le pillage des ressources naturelles mais le transport des déchets vers l'Afrique, et ainsi de pollution (Heger 2017). En ce qui concerne la politique coloniale israélienne, elle implique et le pillage des ressources et le transport des déchets. Il ressort clairement des attitudes des militants écologistes à l'intérieur des colonies israéliennes qu'Israël a fait des colonies juives dans les territoires palestiniens occupés des zones industrielles en dehors de tout cadre juridique respectueux de l'environnement ${ }^{6}$. L'un des rapports de B'Tselem a mis en évidence cette évolution en répertoriant 15 usines israéliennes de recyclage et de traitement de déchets dangereux dans les territoires palestiniens, dont cinq concernent des substances toxiques, cancérigènes, radioactives, potentiellement explosives qui ont bénéficié de facilités juridiques et d'exemptions fiscales :

Sewage sludge: Compost Or Factory Ltd., the northern Jordan Valley

Medical waste: Eco Medical Ltd., Ma'ale Efrayim Industrial Zone

Solvent waste: MTA Recycling Technologies Ltd., Mishor Adumim Industrial Zone

Oil waste: Green Oil Energy Ltd., West Ariel Industrial Zone 
Metal, electronics, and battery processing: EMS Refiners of Precious Metals Inc., Shilo Industrial Zone.

L'un des phénomènes les plus manifestes de la colonisation toxique en Palestine est ce qui se passe dans la région d'Idna, près d'Hébron, où certains groupes de la société palestinienne locale transforment et vivent des déchets produits soit en Israël ou dans les colonies. Ce qui caractérise la structure coloniale est la capacité à affecter tous les aspects de la vie quotidienne des peuples colonisés en leur imposant des contraintes qui sont légitimées par une classification sociale ou une idéologie de racisme. Le terme de colonialité permet d'aller au-delà du discours de symétrie, en privilégiant une approche par le bas, basée sur la vie quotidienne des groupes dominés et vulnérables que sont les Palestiniens, et en particulier, les bédouins de la zone C. Ces groupes mènent une lutte existentielle contre l'occupation coloniale israélienne qui vise leur disparition au moyen de déplacements, de transferts et d'expulsions.

\section{Résister c'est rester}

Dans la zone C qui est riche en ressources naturelles, en terres cultivables, en espaces verts et en ressources en eau, vivent environ 300000 Palestiniens. Outre les habitants dans les zones limitrophes des grandes villes comme Jérusalem, Hébron et El Birah et les habitants des villages de la vallée du Jourdan, on trouve 183 communautés bédouines dans la zone $C$. Les communautés bédouines sont les communautés les plus pauvres de la société palestinienne. Ils vivent surtout de l'élevage, et certains d'entre eux, également de l'agriculture. C'est pourquoi il est si important pour eux d'avoir accès aux ressources naturelles et aux terres. Ces droits ancestraux sur ces terres, qui sont liés au mode de vie spécifique de cette population autochtone, doivent être respectés. Parmi les villages palestiniens présents en zone $C$, seulement 16 ont obtenu l'approbation de leur "masterplan". Le masterplan est une condition préalable pour l'obtention de services tels que la construction d'écoles, l'assainissement, électricité, etc. De plus, sans ce plan directeur, les communautés bédouines sont susceptibles d'être expulsées de leurs maisons, de voir leurs fermes et leurs réservoirs d'eau détruits, et d'être déplacées en vue de s'approprier leurs terres.

J'ai mentionné précédemment le cas de la communauté de Wadi Saab qui est une communauté de réfugiés, mais nous pouvons évoquer aussi d'autres cas comme la communauté du Khirbet Tell el Himma qui sont des déplacés internes. Ce village a été détruit lors de la guerre de 1967, mais certains de ses habitants ont décidé de revenir et d'habiter à nouveau sur les terres de leurs ancêtres. L'un d'eux, appelé Abu Mahyoub, m'a confié que son père est en effet enterré à quelques mètres de sa maison ${ }^{7}$. À Khirbet Tell el Himma, 130 personnes vivent actuellement de l'agriculture et de l'élevage de chèvres et de vaches. L'enregistrement au cadastre et le titre de propriété de leur terre (400 Dunums) remontent à la période ottomane. En 1968, les autorités israéliennes leur ont proposé une réinstallation, mais ils ont refusé de quitter leur terre. La communauté du Khirbet Tell el Himma fait l'objet d'un harcèlement continu de la part de l'administration civile israélienne et des colons de Mahoula destiné à les faire partir. Depuis 2009, quatre incidents de démolitions des maisons ont eu lieu aboutissant au déplacement de 50 personnes dont 21 enfants. ${ }^{8}$

$\mathrm{Du}$ point de vue des droits humains, les États doivent adopter des mesures pour garantir que les groupes vulnérables ne soient pas victimes de discrimination. Par 
exemple, ils devraient adapter leurs politiques de services à ceux qui ont le plus besoin d'assistance, et ne pas cibler uniquement les groupes majoritaires. La puissance occupante a le devoir de fournir des services et des infrastructures, y compris l'eau et l'électricité, à la population locale, ce qu'elle ne fait pas.

Pour décrire l'occupation israélienne, Human Rights Watch propose dans son rapport de 2010 quatre éléments dont le premier est la discrimination raciale. Ces discriminations raciales structurent la législation, l'exploitation des ressources naturelles et l'exploitation de la force du travail des palestiniens. De même, John Dugard et John Reynolds considèrent la colonisation israélienne comme une classification sociale fondée sur la race. Ils écrivent que même si la pratique de classification sociale ne repose pas explicitement sur le terme race, il est utile de l'utiliser pour comprendre et lutter contre les formes de discrimination et de brutalité de l'occupation israélienne.

Les identités juive et palestinienne, bien qu'elles ne soient généralement pas considérées comme des "races" au sens ancien (discrédité) des catégories biologiques ou de couleur de peau, sont construites comme des groupes se distinguant par leur ascendance ou leur descendance ainsi que par leur appartenance ethnique, nationalité et religion. En tant que tels, ils se distinguent les uns des autres sous un certain nombre de formes dans le cadre des paramètres de discrimination raciale en vertu du droit international des droits de l'homme (Dugard, Reynold 2013 : 889).

Il faut y ajouter que dans le domaine de l'accès aux ressources en eau dans les territoires palestinien occupés le rapport présenté par le député socialiste Jean Glavany à l'Assemblée Nationale française en 2010 n'a pas hésité à parler d'apartheid. ${ }^{9}$

La loi de Régularisation consacre un système d'apartheid où les intérêts d'un groupe, les colons, prévaut et domine au détriment de ceux des Palestiniens qui sont des personnes protégées selon le droit humanitaire. En Novembre 2017, le parlement israélien vote en majorité en faveur d'une loi dont l'objectif est d'assurer le maintien et le développement de la colonisation israélienne dans les territoires palestiniens occupés (la loi de la régularisation). La Knesset légifère pour la première fois sur une question ne relevant pas du territoire israélien. Dans le contexte de l'occupation militaire de la Cisjordanie, c'est en effet le commandement militaire qui détient le pouvoir de légiférer selon le droit international humanitaire. En votant la loi de dite de régularisation des colonies israéliennes en Cisjordanie, le Parlement israélien exerce une souveraineté sur un territoire qui n'est pas sous sa juridiction. Ce qui est considéré comme un acte d'annexion, un acte illégal selon le droit international.

La loi de Régularisation de 2017 va même à l'encontre de la politique de la HCJ menée depuis sa décision Dweikat contre le Gouvernement Israélien 390/79 1979 : « selon notre système juridique le droit de propriété individuel est une norme juridique fondamentale dans le droit pénal comme dans le droit civil... ». Cette position a été réaffirmée dans la décision de la colonie d'Amona 9949/08 2014. Il est à souligner que $30 \%$ des colonies ont été établies sur des terres privées palestiniennes ${ }^{10}$. La loi de Régularisation est en violation de l'article 56 de la Convention de La Haye : "Private property cannot be confiscated » et de l'article 8(2)(a)(iv) du traité de Rome 1998. La loi propose de confisquer des terres privées pour qu'elles soient par d'autres personnes sans que cela ne corresponde à des nécessités militaires ou publiques. La loi propose également de payer les propriétaires des terres ou bien de leur imposer de s'installer en 
échange sur d'autres terres mais il faut rappeler ici la jurisprudence de la Cour de Nuremberg:

The payment of a price or other adequate consideration does not; under such circumstances relieve the act of its unlawful character. Similarly, where a private individual or a juristic person becomes a party to unlawful confiscation of public or private property [...].

Ces violations du droit de la propriété ont également pour conséquences des violations du droit à l'emploi et à une vie digne. Dans ses recherches sur le transfert des déchets vers la région d'Idna/ Hébron, Ya'akov Garb souligne que le nombre de frets routiers à destination et en provenance de la zone sud de la Cisjordanie est bien supérieur à ce que les autorités officielles israéliennes savent et autorisent. Selon ces autorités, le nombre de camions, y compris ceux qui transfèrent de déchets, est de 600 par jour alors que l'étude de Ya'akov Garb a trouvé que ce nombre est de 3361 camions par jour. "The overall quantity of trucks was also much larger than expected: 3361 trucks in the 24hour period" (Garb 2015: 100).

L'étude de Ya'akov Garb est extrêmement importante, et les conclusions qu'il soutient sont, à mon avis, plus étroitement liées à la thèse sur la criminalité du parti dominant et puissant. Il voit la différence entre les attentes des autorités d'occupation et ce qui se passe sur le terrain, ce qui, selon lui, constitue une lacune dans le système de contrôle et de manipulation (ibidem : 106). Ce qui se passe sur le terrain reflète ce que Gregg Barack a affirmé, à savoir que l'État, à travers des politiques formelles et informelles, s'engage non seulement dans le contrôle de la criminalité, mais aussi dans le développement de sa criminalité et celle des autres. Selon lui, l'Etat est criminogène (Barak 1990 : 12).

\section{La pertinence du droit international de l'environnement dans le contexte de la colonisation toxique}

La pertinence du droit international de l'environnement dans le contexte de la colonisation toxique s'appuie sur l'évolution de ce droit en un droit pénal de l'environnement en particulier en ce qui concerne le transfert des matières toxiques et dangereuses et sur ce qu'il implique comme critique à la centralité de l'Etat et sa responsabilité dans le système juridique international. Il favorise d'une part l'élargissement des droits aux autres sujets que l'Etat et d'autre part l'adoption d'une conception de responsabilité comprenant à la fois la responsabilité individuelle et collective.

Les crimes commis dans les territoires palestiniens occupés par l'État israélien et ses citoyens sont des atteintes à des obligations des "règles premières" sous des traités internationaux sur la pollution provenant du transfert des matières toxiques. Il s'agit aussi des crimes qui se produisent dans un contexte de colonisation. Je pense que Doris Farget a raison de privilégier le rapport entre environnement et colonialisme vu la reproduction comme elle le dit des rapports coloniaux dans le domaine de l'environnement et ses effets sur les droits des peuples indigènes (Farget 2016). A mon avis, la dichotomie modernité/colonialité permet et de privilégier la question de la colonisation toxique et d'insister sur la forme que peut prendre cette dichotomie à savoir une opposition entre un Etat et un peuple colonisé et indigène. Il est à noter que malgré la centralité de l'Etat dans le système juridique international, le droit 
international de l'environnement et en raison de son lien avec les droits de l'homme a montré une certaine sensibilité envers les droits des peuples indigènes et colonisés qu'il faut supporter et renforcer ${ }^{11}$. Dans un tel contexte, la lutte d'un peuple colonisé et indigène comme le peuple palestinien ne peut être que tiraillée entre une logique d'intégration où l'accent est mis sur la normativité internationale et une autre logique critique et globale qui s'inscrit dans la démarche voulue par les théoriciens de la colonialité, en insistant sur la résistance d'un peuple indigène contre une structure inégale et violente. La Palestine est vue comme un microsome de cette lutte globale pour la justice comme le dit John Collins.

En ce qui concerne la responsabilité sur les crimes environnementaux, elle est à considérer comme étant à l'intervalle entre la responsabilité individuelle et collective. D'une part, il y a une responsabilité de la part des individuels dans un régime démocratique pour la continuité de la structure de domination (Finkelstein et al. 1994), et d'autre part la responsabilité de l'Etat s'appuie sur le fait que L'Etat légifère et tolère des activités polluantes de la part des acteurs privés endommageant les intérêts d'autres peuples.

\section{Redéfinir la légitimité de l'Etat sur le plan international}

En matière de pollution, la Palestine a adhéré à plusieurs traités internationaux parmi lesquels se trouvent trois traités environnementaux sur la pollution : la Convention de Bâle de 1989, entrée en vigueur en 1992, la Convention de Rotterdam de 1998, effective en 2004, et enfin la Convention de Stockholm de 2001, appliquée depuis 2004. Il faut dire que les conflits ne suspendent pas l'application des traités sur l'environnement, ce qui signifie que ces traités doivent être interprétés conformément au droit des conflits et de l'occupation, qui comprend le droit international humanitaire et les droits humains. Selon le principe 23 de la Déclaration de Rio de 1992, l'environnement et les ressources naturelles d'un peuple sous occupation et sous contrôle étrangers doivent être respectés.

Le Secrétariat de la convention de Bâle joue un rôle important dans l'échange d'informations et de risques entre les Etats membres. Selon l'article 19 de la ladite Convention, le Secrétariat peut être saisi par un État si cet État estime qu'un autre État partie a agi en violation de la Convention. C'est ce qui a été activé par la partie palestinienne en ce qui concerne l'arrêt du transfert de déchets dangereux vers les territoires palestiniens. Le Secrétariat a été informé de la saisie de camions transportant des déchets et des matières dangereuses vers la région d'Hébron ${ }^{12}$. Les Palestiniens ont réussi à faire intervenir le Secrétariat dans le conflit concernant le transfert des déchets d'un côté à l'autre de la Ligne Verte. Par-là, les Palestiniens ont fait reconnaître aux autorités israéliennes que son interlocuteur dans la solution de ces problèmes n'est pas l'Administration civile de l'occupation israélienne, mais le gouvernement israélien et plus précisément l'Autorité israélienne pour l'environnement. Cela veut dire, utiliser la convention de Bâle pour insister sur un rapport interétatique et non pas d'occupant/occupé et sur l'existence des frontières entre Israël et les Territoires palestiniens occupés. L'intervention du Secrétariat a interdit à la partie israélienne de recourir à la force pour libérer les personnes arrêtées par les Palestiniens pour leur implication dans le trafic de déchets dangereux. 
31 Les Palestiniens pourraient renforcer leur position internationale en introduisant au sein du droit palestinien une distinction entre le crime de transfert interne d'une substance dangereuse et le crime d'importation de telles substances, ce qui n'est pas clair dans le texte de la loi palestinienne de 1999. Et ils pourraient même aller plus loin en introduisant la compétence extraterritoriale comme c'est le cas en Allemagne. Au paragraphe 3 de l'article 4 de la Convention de Bâle, « les États parties considèrent le transfert de déchets comme un crime ", mais cela n'entraîne pas en effet la possibilité de poursuites internationales. Cette lacune a été prise en compte par certains États parties, comme l'Allemagne, dont le droit pénal (section 2, article 326, 1994) introduit la possibilité de poursuites au-delà de ses frontières.

Ceci évoque la logique d'intégration au sens d'Emile Durkheim. La logique d'intégration permet de distinguer entre une conduite normale et une autre pathologique. Dans son ouvrage sur l'Allemagne en 1915, Emile Durkheim évoque la volonté de puissance de l'Allemagne qui à mon avis s'applique aujourd'hui à l'Etat d'Israël. Il écrit que pour les Allemands il était impossible de concevoir l'Etat sans la guerre et son droit à conduire une guerre constituait la principale caractéristique de sa souveraineté. Et d'ajouter que lorsque l'État est incapable de porter l'épée, il ne mérite pas son nom (Durkheim 1915). A la différence de l'Allemagne d'hier, l'Etat d'Israël justifie ses actions militaires non pas par le principe de la souveraineté mais par le droit à l'auto-défense. Selon la sociologue franco-israélienne, Eva Ilouz, la direction politique israélienne a, dès le début, lié Holocauste et nationalisme palestinien pour faire de la présence palestinienne une menace existentielle et pour justifier sa guerre continue contre le peuple palestinien par le droit à l'auto-défense (Ilouz 2020).

33 L'intégration de la Palestine dans la société des Etats (l'ONU, la convention du Rome ou autre) est un pas important pour assurer les droits des palestiniens à la dignité et au développement. La décision rendue par la chambre préliminaire du Tribunal pénal international attestant de sa compétence pour enquêter et statuer sur les crimes commis dans les Territoires palestiniens occupés aurait été impossible sans cette logique d'intégration que l'autorité palestinienne n'a cessé de mettre en œuvre en travaillant à obtenir une large reconnaissance de leur Etat ${ }^{13}$. Toutefois cette intégration a ses limites. Tout d'abord, il n'y a aucun changement dans la vision israélienne du conflit, comme elle a très bien décrit Eva Ilouz. Au contraire, la société israélienne constate même qu'une politique de la force est payante comme le montrent les accords récemment signés avec les pays arabes du Golfe. Par ailleurs, il est peu probable que les dirigeants politiques israéliens changent leur vision des Palestiniens, qui sont considérés comme des ennemis. Le concept d'intégration s'appuie en outre sur des normes internationales pour lesquelles la légitimité de l'Etat n'existe que dans un contexte interétatique. Il faudrait donc reconsidérer ces normes pour tenir compte des revendications des peuples colonisés et indigènes, et de ce qu'elles impliquent pour la légitimité de l'Etat dans le cadre du droit international.

L'avantage du concept de colonialité réside justement dans le fait qu'il met en exergue la lutte d'un peuple indigène contre une structure coloniale. Il implique une critique de la modernité dont l'Etat constitue une des formes. L'Etat est une forme moderne au moyen duquel s'exerce la colonisation et c'est au nom de l'Etat et de sa souveraineté que l'on continue à nier le droit à l'autodétermination de l'indigène. Il n'est pas étonnant que le juriste israélien Tal Becker parle de la relativité du droit à l'autodétermination du peuple palestinien vis-à-vis la souveraineté de l'Etat d'Israël 
(Becker 1998). S'appuyant sur le nationalisme, l'Etat ravive et modernise "les guerres privées" de la période féodale contre les minorités et les peuples indigènes (Tolstykh, Aasi 2020 :71).

Dans le rapport de la Commission du Juge Théodore Or, les Palestiniens de l'intérieur d'Israël sont décrits comme une "homeland minority" et/ou une minorité indigène ${ }^{14}$. Cette Commission a précisé ce qu'elle entendait par indigénéité en mentionnant notamment le fait que cette communauté palestinienne était présente avant la création de l'État et le processus par lequel elle est devenue un groupe non-dominant et minoritaire dans la société israélienne. Ces éléments sont inclus dans la définition des groupes indigènes du rapporteur spécial des Nations unies sur la discrimination à l'encontre des populations autochtones par l'ancienne Commission aux droits de l'homme, José Martinez Cobo (nommé en 1971), sauf l'élément qui concerne le lien entre l'existence de ces groupes et le fait qu'ils se retrouvent dans des situations de colonialisme interne ou de colonialisme de peuplement (1986) (Keal 2003 :7). Cet élément est au contraire crucial dans la définition proposée par le sociologue mexicain, Rodolfo Stavenhagen, pour lequel le terme indigenismo indique la domination d'un peuple européen sur un peuple non européen et la perte de sa souveraineté sur son territoire et sur ses ressources naturelles en devenant un groupe minoritaire dans la société du colonialisme de peuplement.

the discourse of indigenousness leads to a denunciation of injustices (and crimes)

committed against indigenous peoples (genocide, plunder, servitude, discrimination) and to the formulation of specific rights that derive from the injustices suffered (Lam 2000: 4).

Il est à noter que le droit international défavorise les peuples indigènes en ce qui concerne le droit à l'auto-détermination. En limitant le droit à l'autodétermination aux peuples de territoires non-indépendants selon l'Article 73 de la Charte de l'Onu, la pratique internationale privilégie la question du territoire comme préalable à l'exercice du droit à l'autodétermination dans son lien à la question de la souveraineté et permet aux Etats de décider quel groupe peut faire valoir son droit à l'autodétermination (Summers 2005). De même, professeur James Anaya, de la Faculté du Droit à l'Université de Colorado, attire notre attention sur le fait que même dans le traité de l'Organisation International du Travail N0.169 (1989), la notion de peuple n'a pas la signification qu'elle a habituellement dans le droit international. Selon lui, en l'état, le droit international actuel exclut plusieurs groupes et ne prend pas en compte leurs droits à l'autodétermination (Anaya $2004: 80-81$ ).

Le sociologue australien Tim Rowse est ainsi en faveur d'un retour au droit naturel, à partir de la centralité des valeurs morales des droits humains. Pour ce dernier, « un Etat illégitime moralement est soit un Etat dont les origines sont liées au pillage des biens de peuples autochtones et dans lequel les descendants de ces peuples continuent à en souffrir considérablement, soit un Etat dans lequel ont été perpétuées des pratiques racistes qui menacent l'existence des peuples autochtones" (Keal 2003 :141). Ces propos renvoient à la dimension structurelle moralement condamnable de configurations étatiques où l'idéologie raciste est omniprésente, mais nous invitent aussi à prendre en compte la continuité de cette structure et ses implications en termes du pillage des ressources naturelles et de destruction de l'environnement palestinien. faut tenir compte lorsque nous abordons les crimes environnementaux et tout 
particulièrement quand la situation a perduré et s'inscrit dans la continuité (Nollkaemper 2010 : 326).

\section{La responsabilité environnementale entre individuel et collectif}

En matière des crimes environnementaux, deux positions coexistent. La première est celle de la Cour de cassation française dans son arrêt datant du 25/03/1981, par lequel elle a condamné un directeur d'entreprise et un responsable des ouvriers pour leur négligence concernant la pollution des eaux fluviales par un employé (Littman-Martin 1982 : 158). Dans cette décision, la Cour a prêté attention à la hiérarchie des responsabilités et des pouvoirs pour déterminer la responsabilité des dommages environnementaux, c'est-à-dire qu'elle a privilégié la responsabilité à partir de la notion d'intention. Alors que dans l'accord de 1990 de la Commission Economique des Nations Unies pour l'Europe relatif à la responsabilité civile pour les dommages survenus lors du transport de matières dangereuses par voie routière, ferroviaire ou de navigation intérieure (CRTD), la responsabilité stricte et limitée a été adoptée. En se concentrant sur le résultat du délit environnemental on privilégie la responsabilité objective et la responsabilité de l'Etat dans le contexte international.

Bien que marginalisée au cours du $\mathrm{XX}^{\mathrm{e}}$ siècle, qui s'est concentré sur le principe de la responsabilité pénale individuelle, la responsabilité collective connaît un certain regain d'intérêt grâce à la montée en puissance du droit de l'environnement. Accordant une priorité au traitement des dommages sans nécessairement se pencher sur la source de l'action, qu'elle soit le fait du secteur public ou du secteur privé, aboutit à privilégier la responsabilité des États en matière d'environnement.

Pour certains, la position du tribunal de Nuremberg stipulant que le droit pénal s'applique uniquement aux individus conduit à occulter la dimension collective des crimes internationaux qui impliquent une action et une responsabilité collectives (Fletcher 2002 : 1514 ; Fletcher, Ohlin 2005 : 542-544). Les crimes internationaux y compris les crimes environnementaux en tant que crimes de guerre sont des crimes individuels associés à la culpabilité morale collective. Développé par l'Organisation de coopération et de développement économiques (OCDE), confirmé dans le principe 16 de la Déclaration de Rio de 1992, et mentionné par la Convention de Stockholm, le principe de "pollueur-payeur" paraît un principe en accord avec l'approche libérale et individuelle du droit pénal. C'est pourquoi, il est inapplicable en ce qui concerne l'entité étatique. Toutefois, à mon sens, ce principe peut tout à fait être étendu au-delà de l'individu au secteur industriel et à la société de l'État occupant. Le bénéficiaire ultime des entreprises de traitement des déchets dans les Territoires palestiniens occupés est la société israélienne. Ce qui implique qu'il est nécessaire de se pencher sur la responsabilité des individus et des entreprises, mais aussi de l'occupation en tant que structure productrice de crimes contre l'environnement (criminogène).

Les actes individuels ne se font pas dans un vide, ils s'inscrivent dans une action collective et sont structurés par celle-ci. Je trouve que l'usage fait par André Nollkaemper du concept de "crime systémique" développé par B.V.A Röling est ici très approprié. Par ce concept, on entend que 'les gouvernements donnent l'ordre de commettre des crimes ou les encouragent ou les favorisent ou les tolèrent'. Ces crimes servent le système et sont causés par le système' (ibidem : 316). Ce qu'André 
Nollkaemper décrit comme un 'climat normatif prévalant au sein du système', c'est-àdire impliquant l'autorisation large latente d'actes de violence par des formes de propagande ou/et de déshumanisation de l'autre, de la victime, correspond parfaitement bien aux propos de Gershin Baskin:

The settlement project in the West Bank is nothing less than an act of national suicide. The most significant and dangerous obstacle in our ability to reconcile peace with our neighbors is the continuation of the adherence to the archaic modes of our yearning for Zion expressed by settling the hilltops surrounding Palestinian towns, villages and cities that turn the lives of Palestinians into a daily hell (Baskin 2007).

Le concept du crime systémique est compatible avec l'idée de criminalité de la partie dominante, ce qui signifie que le préjudice peut provenir directement de l'État ou d'autres acteurs dont les actions ne peuvent être séparées de la structure de contrôle existante. C'est à cela que tentent de se référer certaines études (Zeitoun, Warner 2006) et rapports internationaux ${ }^{15}$ et locaux ${ }^{16}$ sur la question palestinienne de l'environnement en se concentrant sur l'anti-impérialisme et sur le concept de justice.

D'après certaines critiques, le droit international de l'environnement favorise le retour à la théorie traditionnelle de la criminologie qui se concentrait sur l'influence de la structure et considérait que l'État est une institution qui contrôle la criminalité et la génère (Ross 1998). Toutefois, à mon sens, il ne s'agit pas simplement d'un retour à la théorie traditionnelle de la criminologie. Ce que nous voyons c'est l'émergence d'une théorie nouvelle qui favorise la complémentarité entre les deux formes de responsabilité en matière des crimes environnementaux. A l'instar d'André Nollkaemper je défends l'idée qu'il n'y a pas d'alternative à la responsabilité individuelle et qu'il faut l'aborder comme une sanction contre le groupe dans le cadre d'une politique de réparation (ibidem : 336-337). Ce qui est nouveau dans le passage des conventions de Genève de 1949 à la convention de Rome de 1998 c'est que les individus ne peuvent plus se protéger derrière la responsabilité collective de l'Etat.

En septembre 2018, le Procureur du Tribunal Pénal International a confirmé la compétence du Tribunal sur les affaires environnementales, ce qui ouvre bien entendu la porte à des poursuites pour les violations de l'environnement palestinien par l'occupation israélienne. Il s'agit d'actes d'anéantissement environnemental, du vol de ressources naturelles (pillage) et l'exploitation de l'économie locale au profit de l'occupation (spoliation), de même que le transport et le stockage illégal de déchets.

La qualification d'un crime environnemental comme crime de guerre doit être fondée sur ce qui a été spécifié dans les articles du Protocole additionnel I 1977 (Article 35, paragraphe 3 et Article 55), c'est-à-dire qu'un niveau grave, répandu et durable de dommage environnemental doit être prouvé. Le rapport fait à la demande du procureur du Tribunal pénal international pour l'ex-Yougoslavie à la suite des bombardements de la Yougoslavie par les forces de l'OTAN en 1999 les a pour ce motif mis en cause. Le rapport a été publié, mais l'identité du groupe d'experts qui l'a rédigé n'a pas été divulguée. La conclusion la plus importante de ce groupe d'experts est qu'il considère la protection de l'environnement comme faisant partie de la protection de la population civile (Bothe 2001).

Il est à souligner que le droit pénal environnemental s'adresse principalement aux entreprises responsables de la production et de la distribution de matières dangereuses. D'une part, on trouve que dans certains systèmes juridiques nationaux on 
considère que les entreprises n'ont pas la capacité de décider car elles ne sont pas un être rationnel et moral responsable de ses actes. La loi allemande ne considère pas les entreprises puissent commettre des délits. De même, certains juristes privilégient le "soft law" en tant qu'une juridicité sans contrainte et au sens de la moralisation où les entreprises économiques peuvent offrir une culture éthique qui n'autorise pas les violations du droit international (Osman et al. 2018 :4). Récemment, les Nations Unies ont publié une liste de 500 entreprises étrangères et israéliennes dont les activités participent au développement des infrastructures dans les colonies juives en Cisjordanie. Le Haut-Commissaire aux droits de l'homme a envoyé une enquête à ces entreprises pour leur demander de cesser de participer à la violation du droit international. Cette étape s'inscrit dans une approche de "soft law" et soulève un principe moral pour obliger les entreprises à respecter le droit international.

D'autre part, il y a ceux qui exigent, au contraire, un changement de paradigme en ce qui concerne la question des crimes de guerre commis par des entreprises et des institutions économiques afin qu'elles puissent être considérées comme responsables de leurs actes tout comme les personnes physiques. La responsabilité criminelle des entreprises économiques a été écartée par le traité de Rome 1998. Ce qui fait que le Tribunal Pénal International ne peut que punir les directeurs ou les responsables de ces entreprises selon le précédent de Nuremberg et non pas les entreprises elles-mêmes. Michael A. McGregor a montré que vu le rôle prépondérant des institutions économiques dans le pillage des ressources naturelles des peuples indigènes, il est crucial d'aller au-delà du précédent de Nuremberg pour lancer des poursuites contre ces institutions économiques (McGregor 2009 : 490-492), dans le cadre international ou extraterritorial.

\section{Conclusions}

L'environnement est un domaine privilégié pour montrer les dispositifs de domination/exploitation décrite par le terme de colonialité du pouvoir dans les Territoires palestiniens occupés. Les positions des activistes environnementaux dans les colonies ainsi que l'analyse de Ya'akov Garb montrent bien la responsabilité de l'Etat occupant, ce qui exige une attention particulière à la dimension collective des actes criminels individuels. Il faut tenir compte du rôle de l'Etat ou de la partie dominante dans le conflit dans l'autorisation et la génération des crimes. Le droit international de l'environnement, tout en favorisant une certaine conception collective de la responsabilité, ne peut que conduire à l'émergence d'une criminalité environnementale où responsabilité individuelle et collective sont prises en compte.

Qui plus est, accorder une compétence extraterritoriale en matière de dommages environnementaux aux juges palestiniens exigerait le développement du droit environnemental palestinien mais aussi un changement d'attitude de la part de la direction politique ainsi que de l'opinion publique palestinienne envers les juges palestiniens en matière d'interprétation. Il n'est pas raisonnable d'adhérer à des traités internationaux en matière environnementale et de s'abstenir de les publier dans le journal officiel. En outre, le texte de la loi palestinienne sur l'environnement N0.7 (1999) considère que les traités internationaux sont complémentaires de la loi nationale (l'Article 77), ce qui signifie que le juge national peut s'appuyer sur ces traités 
internationaux pour interpréter la loi palestinienne sur l'environnement (le principe de l'efficacité).

\section{BIBLIOGRAPHIE}

Alleson I., Levin J., Brenner Sh., Al Hmaidi M. S., 2013 "Peace and Pollution : An Examination of Palestinian-Israeli Trans-Boundary Hazardous Waste Management 20 Years after the Oslo Peace Accords", in Journal of Peacebuilding \& Development, 8, 1: 15-29.

Anaya J., 2004 "Developments within the modern era of human rights, ch.3, Self- determination: A foundational principle", in Anaya J., Indigenous peoples in International law, Oxford, Oxford University Press.

Barak G., 1990 "Crime, Criminology and Human Rights: Towards an Understanding of State Criminality", The Journal of Human Justice, Vol. 2, N. 1, Autumn.

Baskin G., 2007 "Why A Am A Neo Zionist?" Jerusalem Post, July 3.

Becker T., 1998 "Self-determination in perspective: Palestinian claims to statehood and the relativity of the right to self-determination", Israel Law Review, Vol.32, n. 2: 301-354.

Bothe M., 2001 "The protection of the civilian population and NATO bombing in Yugoslavia: Comment on a Report to the Prosecutor of the ICTY", European Journal of International Law, vol.12, N. 3: $531-535$

Collins J., 2010 "Between Acceleration and Occupation: Palestine and the Struggle for Global Justice", Studies in Social Justice, Volume 4, Issue 2: 199-215.

Dugard J., Reynold J., 2013 "Apartheid, International Law, and the Occupied Palestinian Territory", The European Journal of International Law, Vol. 24, N. 3: 867-913.

Durkheim E., 1915 “ L'Allemagne au-dessus de tout ”, La mentalité allemande et la guerre, Paris, Armand Colin (collection « Études et documents sur la guerre »).

Farget D., 2016 « Colonialisme et pollution environnementale : prolongement et effets sur les droits des peuples autochtones ", Criminologie, 49 (2) : 95-114.

Finkelstein G. N, Depret A., Passevant Ch., 1994 « Réflexions sur la responsabilité de l'État et du citoyen dans le conflit arabo-israélien », L'Homme et la société, N. 114 : 37-50.

Fletcher G., 2002 “The Storrs Lectures: Liberals and Romantics at War: The Problem of Collective Guilt", The Yale Law Journal, Vol.111: 1499-1573.

Fletcher G.P., Ohlin J.D., 2005 Reclaiming Fundamental Principles of Criminal Law in the Darfur Case, Cornell Law Faculty Publications, Paper 435: http://scholarship.law.cornell.edu/facpub/435

Garb Y., 2015 "Porosity, Fragmentation, and Ignorance: Insights from a study of freight traffic", in Stéphanie Latte Abdallah and Cédric Parizot (eds.), Israelis and Palestinians in the Shadows of the Wall: Spaces of separation and occupation, Surrey and Burlington, Ashgate, Border Regions Series, Ch. 5: 89-107.

Gathii J.T., 2006-2007 “Imperialism, Colonialism, and International Law”, 54 Buff. L. Rev. 1013. 
Guha R., Martinez-Alier J., 1997 Varieties of Environmentalism: Essays North and South, Earthscan Publications Ltd, London.

Heger M., 2017 “Trafficking in Hazardous Wastes (Article 28L) and Illicit Exploitation of Natural Resources" (Article 28Lbis), in G. Werle, M. Vormbaum (eds.), The African Criminal Court, International Criminal Justice, Series 10, Chapter 8: 125-136.

Heller Sh., 2019 "The plan to set up a waste recycling site in Maale Adumim is underway - area residents are furious”, in Kol HaHair, Jerusalem, 23/10/2019 www.kolhair.co.il/jerusalem-news/ maale-adumin/110822.

Ilouz E., 2020 « Comment le mensonge a envahi Israël », Haaretz, 16 Décembre.

Keal P., 2003 European Conquest and the Rights of Indigenous Peoples, The moral backwardness of international society, Cambridge Studies in International Relations.

Lam M.C., 2000 At the edge of the State: Indigenous peoples and self-determination, Ardsley, NY, Transnational Publishers, Inc.

Littman-Martin M.J. 1982 « Droit pénal de l'environnement », Revue Juridique de l'Environnement : 154-169.

Levy N., 2009 Transboundary Environmental management under asymmetry, Hebrew University / Department of Geography. May (in Hebrew).

Marks S., 2008 "Exploitation as an international legal concept”, in Susan Marks (ed.), International Law on the Left: Re-examining Marxist Legacies, Cambridge University Press: 281-308.

McGregor M.A., 2009 “Ending Corporate Impunity: How to Really Curb the Pillaging of Natural Resources”, 42 Case W. Res. J. Int'l L.469, available at: http://scholarlycommons.law.case.edu/jil/ vol42/iss1/46.

Meyer Y., 2013 Director general of Samaria's environmental protection association, Jerusalem Post $17 / 06 / 2013$.

Mickelson K., 2013 "South, North, International environmental law, and international environmental lawyers", in Yearbook of International Environmental Law, Vol. 11: 52-81.

Nollkaemper A., 2010 "Systemic Effects of International Responsibility for International Crimes", 8 Santa Clara J. Int'l L. 313, available at: http://digitalcommons.law.scu.edu/scujil/vol8/iss1/12.

Osman F., Prince H. A., Rigaud M.-C., 2018 Soft law et droit du commerce international, Contribution à l'étude du renouvellement des sources du droit, Colloque International, Montréal, 11 et 12 mai 2017, Lexis Nexis Canada Inc.

Pouchepadass J., 1993 " Colonisations et environnement », Revue française d'histoire d'outre-mer, tome 80, n. $298,1^{\text {er }}$ trimestre : 5-22.

Quijano A., 2007/3 « ‘Race’ et Colonialité du Pouvoir », Mouvements, n. 51 : 111- 118.

Quijano A., 2000 Coloniality of Power, Eurocentrism, and Latin America, Nepantla, Views from South 1.3, Duke University Press: 533-580.

Ross J.I., 1998 "Situating the academic study of controlling state crime", Crime, Law \& Social Change, 29: 331-340, Kluwer Academic Publishers.

Strawson J., 2002 "Reflections on Edward Said and the Legal Narratives of Palestine: Israeli Settlements and Palestinian Self Determination", Penn State International Law Review, Vol. 20: No. 2, Article 4, available at: http://elibrary.law.psu.edu/psilr/vol20/iss2/4. 
Soto P. D, 2009/3, « Modernité et colonialité du pouvoir, du savoir et de l'être », Cahiers de l'Amérique Latine, Philosophie de la Libération et tournant décolonial, 62 : 47-58.

Summers J.J., 2005 “The Right of Self-Determination and Nationalism in International Law”, International Journal on Minority and Group Rights, Vol. 12, n. 4: 325-354.

Tilley V., (ed.) 2011 Beyond Occupation: Apartheid, Colonialism, and International Law in the occupied Palestinian territories, London, Pluto Press.

Tolstykh V., Aasi J., 2020 "Nation Reification or "Nationalizing Nationalism" from the Perspective of International Law”, Russian Law Journal, September, 8(3): 64-83.

Zeitoun M., Warner J. 2006 "Hydro-hegemony - a framework for analysis of trans-boundary water conflicts", Water Policy, 8: 435-460.

Weizman E., 2004 “The Geometry of Occupation", Lecture at the Center of Contemporary Culture of Barcelona (1 March 2004). www.urban.cccb.org.

Yotam B., Tzafrir R., 2018 "Government promotes: Waste from Israeli territories will be recycled in the West Bank", in Haaretz 06/08/2018: www.haaretz.co.il/science/1.6343579.

\section{NOTES}

1. La ligne verte est la ligne du front de l'armistice du 3 avril 1949 et qui sépare l'Etat d'Israël des territoires occupés depuis 1967, les Territoires palestiniens occupés plus le Golan.

2. En 1982, Des habitants de la ville israélienne de Kfar Saba ont réussi à intenter une action en justice contre l'usine de produits chimiques agricoles «Gishuri» pour les dangers qu'il porte à l'environnement. L'usine a rouvert, cinq ans après, dans une colonie industrielle de la ville de Tulkarm en Cisjordanie « Nitsaneh Shalom » (Bourgeon de paix). Depuis lors, les taux de cancer à Tulkarm sont devenus parmi les plus élevés de Cisjordanie.

3. Human Rights Watch Report on "Separate and Unequal, Israel's Discriminatory Treatment of Palestinians in the Occupied Palestinian Territories" December 19, 2010.

4. Dans la décision de la Chambre préliminaire du 05/02/2021 (ICC 01/18), le Tribunal Pénal International a considéré que les normes péremptoires telles que le droit à l'auto-détermination s'imposent aux obligations bilatérales comme les accords d'Oslo (paragraphe $36 \mathrm{pp} .18$ ).

5. Desk Study on the Environment in the Occupied Palestinian Territories UNEP 2003 www.unep.org/download_file.multilingual.asp?FileID=105.

6. «A legislative vacuum exists in West Bank settlements when it comes to the environment, he said. Old environmental regulations seen as applicable to settlements there have been erased and replaced by tougher laws that do not cover Area C, under Israeli civil and military control. As a result, in recent years it has become impossible to force factories in West Bank industrial parks and settlements to comply with environmental restrictions» Yitzhak Meyer, director general of Samaria's environmental protection association, Jerusalem Post 17/06/2013.

7. Visite et rencontre avec les familles du Khirbet Tell el Himmeh le 11/08/2016 dans le cadre d'un projet concernant l'accès des communautés bédouines de la zone $\mathrm{C}$ à l'électricité (la chaire UNESCO de la démocratie et droits de l'homme à l'Université Najah, Naplouse).

8. Weeks after demolition, families from Khirbet Tell el Himma continue to face regular settler and army harassment, (11 November 2016) www.ochaopt.org/content/weeks-after-demolitionfamilies-khirbet-tell-el-himma-continue-face-regular-settler-and-army.

9. Rapport d'information déposé en application de l'article $145 \mathrm{du}$ Règlement par la Commission des Affaires Etrangères à l'Assemblée Nationale en conclusion des travaux d'une mission 
d'information constituée le 5 octobre 2010 sur « La géopolitique de l'eau », Rapporteur M. Jean GLAVANY, Rapport N0. 4070, du 13 décembre 2011.

10. Le rapport du général Baruch Spiegel au Premier Ministre israélien, en 2006, qui offre une banque de donnée sur les colonies, "met en évidence une réalité que l'on tente de cacher depuis des lustres : dans l'écrasante majorité des implantations - plus de $75 \%$-, la construction et/ou l'extension des colonies ont été menées de façon irrégulière. Les travaux ont été réalisés soit sans permis de construire, soit à d'autres fins que celles stipulées dans le permis accordé. Plus grave, de nombreuses infrastructures publiques (routes, écoles, synagogues, séminaires talmudiques, postes de police, etc.) ont été bâties de façon totalement illégale sur des terrains appartenant à des particuliers palestiniens". (Polémique en Israël. Tant de colonie hors la loi... Courrier international 14/04/2009

www.courrierinternational.com/article/2009/04/16/tant-de-colonies-hors-la-loi).

11. Droits de l'Homme et Environnement, Rapport final établi par Mme Fatma Zohra Ksentini, Rapporteur spécial E/CN.4/Sub.2/1994/9, 26 juillet 1994. Ch.III A pp. 25.

12. UNEP-CHW-CAS-TRAF-Palestine. English\%20(2).pdf. Complaint from Palestinian Authority for Environment on Illegal traffic of hazardous wastes to Halhoul town in Hebron region, 19 April 2017.

13. ICC, Pre-Trial Chamber I, SITUATION IN THE STATE OF PALESTINE. No. ICC-01/18, 5 February 2021.

14. Rapport du Comité d'enquête gouvernemental sur les affrontements entre les forces de sécurité et des citoyens palestiniens d'Israël en octobre 2000, vol. 1-27-26 (2003).

15. Rapport d'information déposé en application de l'article 145 du Règlement à l'Assemblée Nationale le 13 décembre 2011 sur « La géopolitique de l'eau » le Rapporteur M. Jean GLAVANY www.assemblee-nationale.fr/13/rap-info/i4070.asp.

16. Environmental injustice in Occupied Palestinian Territory: Problems and Prospects, Report Al Haq 2015 written by Dr. Benjamin Pontin, Vito de Lucia, and Dr. Jesus Gamero Rus.

\section{RÉSUMÉS}

L'article décrit la colonisation israélienne et ses crimes contre l'environnement palestinien. Audelà du processus de paix et du discours de parité, la colonisation du territoire palestinien occupé se faite à un rythme accéléré et intensifié. Dans cette colonisation, les ressources naturelles sont exploitées, et les terres palestiniennes sont transformées en une décharge pour les déchets toxiques. Quelle est la capacité du droit international de l'environnement à prendre en compte les revendications de groupes vulnérables tels que les Palestiniens ? Et quelle conception de responsabilité favorise-t-il?

L'articolo descrive la colonizzazione israeliana e i crimini contro l'ambiente palestinese. Al di là del processo di pace e dei discorsi sulla parità, la colonizzazione dei territori palestinesi procede a ritmo accelerato. Un processo caratterizzato dallo sfruttamento delle risorse naturali e dalla realizzazione di discariche di rifiuti tossici. Qual è la capacità del diritto ambientale internazionale di prendere in considerazione le rivendicazioni di gruppi vulnerabili come $\mathrm{i}$ palestinesi? E quale concezione di responsabilità promuove? 
The article aims to describe the Israeli colonization and its crimes against the Palestinian environment and to question the capacity of international environmental law to take into account the claims of vulnerable groups such as the Palestinians and the State responsibility for environmental crimes. Beyond the peace process and the discourse of parity, the colonization of Palestinian occupied territory is in accelerating and intensifying rhythm. In this colonization, natural resources are exploited, and Palestinian lands are transformed into a tame for toxic and dangerous wastes. It seems that international environmental law provides some means of struggle for Palestinians, but it is still limited when it comes to non-state and non-dominant group rights. Regarding environmental crimes, it seems that international environmental law favors a collective conception of responsibility that must be seen as complementary to individual responsibility.

\section{INDEX}

Mots-clés : colonialité, pollution, droit international de l'environnement, responsabilité collective, Palestine

Keywords : coloniality, pollution, environment international law, collective responsibility, Palestine

Parole chiave : colonialità, inquinamento, diritto internazionale dell'ambiente, responsabilità collettiva, Palestina

\section{AUTEUR}

\section{JONI AASI}

Faculty of Law, Najah University joni.aasi@najah.edu 